\title{
1 year later, 9-13 year olds impaired but not diagnosed were as disturbed as those diagnosed
}

\author{
Angold A, Costello EJ, Farmer EMZ, et al. Impaired but undiagnosed.J Am Acad Child Adolesc Psychiatry 1999 Feb;38:129-7.
}

\section{Question}

What is the outcome of children with psychosocial impairment who do not meet DSM-III-R criteria for any of 29 well defined disorders?

\section{Design}

As part of the Great Smoky Mountain Study, general population screening was done to identify individuals with behavioural and emotional problems. This inception cohort was then followed up for 1 year.

\section{Setting}

11 counties in western North Carolina, USA.

\section{Patients}

A representative sample of 4500 youths aged 9, 11, and 13 years were screened for psychopathology using a subset of items from the Child Behavior Checklist. All children scoring above a predetermined cut off, and a 1 in 10 random sample of those scoring below the cut off were recruited for the study. This resulted in a sample of 1015 participants of whom $90 \%$ were followed up 1 year later.

\section{Assessment of prognostic factors}

A child and primary caretaker were separately interviewed at baseline about the child's psychiatric status, impairment, and service use using the third edition of the Child and Adolescent Psychiatric Assessment (CAPA) which generated DSM-III-R diagnoses, and the Child and Adolescent Services Assessment. The Child and Adolescent Burden Assessment was also completed by parents at the end of the diagnostic interview to measure problems experienced by parents due to their child's emotional or behavioural problems.

\author{
Main outcome measures \\ Diagnosis using the CAPA and other measures of caseness and \\ impairment at 1 year follow up.
}

\begin{abstract}
Main results
At baseline, 140 of the 1015 participants had both a diagnosis and impairment (weighted percentage $7.4,95 \%$ CI $5.5 \%$ to $9.4 \%), 143$ had only a diagnosis but were not impaired $(11.5 \%$, CI $8.7 \%$ to $14.4 \%$ ), and 205 had impairment but no diagnosis $(14.2 \%$, CI $9.8 \%$ to $18.6 \%)$. 135 of the 205 participants with impairment but no diagnosis had a relational problem of which 62 had pure relational impairment unassociated with psychiatric symptoms. Classifying these 62 resulted in weighted population prevalence rates of $1.4 \%$ for sibling relational problems, $3.6 \%$ for parent child relational problems, and $0.6 \%$ for relational problems not otherwise specified. The overall rate of impairing subthreshold disorder was 9.4\%. At 1 year follow up these children were as clinically disturbed as those who met diagnostic criteria for a disorder but were unimpaired. In these subthreshold disorders the most common impairing problems involved oppositional and conduct disorder symptoms (72\%).
\end{abstract}

\section{Conclusion}

After 1 year, children and adolescents who did not meet DSM-III-R criteria for any disorder but who had symptoms associated with psychosocial impairment were as clinically disturbed as those who met diagnostic criteria but were unimpaired.

Sources of funding: National Institute of Mental Health and the Leon Lowenstein Foundation, New York

For correspondence: Dr A Angold, Department of Psychiatry and Behavioral Sciences, Box 3454, Duke University Medical Center, Durham, NC 27710, USA. Fax +1 9196874737.

\section{Commentary}

This study by Angold et al provides important information for clinicians, service providers, and funders. It gives credence to the common clinical practice of using a diagnostic system with an element of flexibility when deciding on who needs service. The finding that impairment is as important as diagnosis in predicting future "caseness," whether that is measured by service use, impairment, diagnosis, perceived problem, or perceived need for help, does suggest the importance of a careful assessment of impairment. Furthermore, it also suggests that alleviating impairment might appropriately be a treatment end in itself.

The most common sources of impairment not connected with a diagnosis are parent-child relationship problems, sibling and peer relationship problems, and "subthreshold" disruptive behaviour problems.
As the authors point out, the rates of relational difficulties make these among the more prevalent childhood problems. Despite this, such relational problems were underrepresented in the group receiving service. Because these relational problems do predict impairment at follow up, attention to them in treatment is clearly warranted, supporting the importance of treating the child in relation to his family as opposed to a more narrow emphasis on the child and his disorder.

The authors argue for including children who are subthreshold for a diagnosis but, with impairment, in the category of severe emotional disturbance. When this is done, the proportion of service users who could then be defined as having a severe emotional disturbance increases from $38.4 \%$ to $59.7 \%$, a substantial shift. This study raises important questions for funding of mental health services for children and adolescents. If both diagnosis and impairment are required for treatment, a much smaller percentage of youth and families in need would qualify for service. Given the predictive validity of impairment itself, service should not be funded solely on the basis of meeting diagnostic criteria.

What the authors do not clarify is the interesting finding that a substantial proportion of individuals receiving service do not meet criteria for either diagnosis or impairment. It might be interesting to examine this group as well as to clarify their reasons for being in the service system.

Susan J Bradley, MD The Hospital for Sick Children Toronto, Ontario, Canada 